\title{
Analysis of plasma termination in the JET hybrid scenario
}

\author{
J Hobirk, M Bernert, P Buratti ${ }^{1}$, C D Challis ${ }^{2}$, I Coffey ${ }^{3}$, P Drewelow ${ }^{4}$, \\ E Joffrin ${ }^{5}$, J Mailloux ${ }^{2}$, I Nunes ${ }^{6}$, G Pucella ${ }^{1}$, T Pütterich, P C de Vries ${ }^{7}$ \\ and JET contributors $\$$ \\ Max-Planck-Institut für Plasmaphysik, Boltzmannstr. 2, 85748 Garching \\ ${ }^{1}$ Unità Tecnica Fusione, C.R. ENEA Frascati, CP65, 00044 Frascati, Italy \\ ${ }^{2}$ CCFE, Culham Science Centre, Abingdon,OX14 3DB, UK \\ ${ }^{3}$ Queen's University, Belfast, BT7 1NN, UK \\ ${ }^{4}$ Max-Planck-Institut für Plasmaphysik, Teilinstitut Greifswald, 17491 Greifswald, Germany \\ ${ }^{5}$ CEA, IRFM, F-13108 Saint-Paul-lez-Durance, France \\ ${ }^{6}$ Associaçāo EURATOM-IST, IPFN - Laboratório Associado, IST, Lisboa, Portugal \\ ${ }^{7}$ ITER Organization, Route de Vinon sur Verdon, 13067 St Paul Lez Durance, France. \\ E-mail: joerg.hobirk@ipp.mpg.de
}

\begin{abstract}
This paper analyses the final phase of hybrid scenario discharges at JET, the reduction of auxiliary heating towards finally the Ohmic phase. The here considered Ohmic phase is mostly still in the current flattop but may also be in the current ramp down. For this purpose a database is created of 54 parameters in 7 phases distributed in time of the discharge. It is found that the occurrence of a locked mode is in most cases preceded by a radiation peaking after the main heating phase either in a low power phase and/or in the Ohmic phase. To gain insight on the importance of different parameters in this process a correlation analysis to the radiation peaking in the Ohmic phase is done. The first finding is that the further away in time the analysed phases are the less the correlation is. This means in the end that a good termination scenario might also be able to terminate unhealthy plasmas safely. The second finding is that remaining impurities in the plasma after reducing the heating power in the termination phase are the most important reason for generating a locked mode which can lead to a disruption.
\end{abstract}


PACS numbers: 52.55.Dy,52.55.Fa, 52.55.Tn, 52.35.Py

Keywords: magnetic fusion, disruptions, termination, hybrid scenario

Submitted to: Nucl. Fusion

\section{Introduction}

Within the last few years significant progress has been made on the hybrid scenario in JET[1]. The hybrid scenario $[2,3,4,5,6,7,8]$ is one of the foreseen ITER scenarios. Compared to the standard 15MA baseline ITER scenario the plasma current is reduced and the normalised beta increased. On JET also the confinement time normalised to the $H_{98, y 2}$-scaling is increased and the scenario is usually obtained by modifying the $q$-profile using a current "overshoot" $[1,9]$. However most of the analysis concentrated so far on the high performance phase of the plasma. Another important aspect is to terminate such high performance plasmas safely. Future devices will have a very limited budget for disruptions before serious damage to the plasma facing components and/or the vacuum vessel and coils could occur. Especially with the introduction of the ITER like wall (ILW) in JET the plasmas often are hampered by impurity accumulation and mode activity when ramping down the power or even before [10]. Earlier a reduced data base has been analysed [11] and preliminary improvements to the termination scenario have been suggested. One aim of this paper is to find the importance of parameters for the termination phase which are in the (far) history of the pulse. This is different to the parameter discussed in [12] where the parameters and their time development during the termination are discussed. The paper will start with a description of a typical hybrid scenario termination. In sections 3 and 4 the database and its analysis are presented. In section 5 a new termination scenario is sketched and an example of a more successful termination is discussed. Then in section 6 the paper is summarised and conclusions are drawn.

\section{Description of an ILW hybrid scenario termination}

In figure 1 time traces of a representative plasma termination are shown. The figure is organised as follows: the top graph contains the plasma current and the magnetic field. The next graph down contains the NBI and ICRH heating power and the total radiated power. In the next graph the radiation peaking is represented as ratios between horizontal central and about half radius line integrated measurements from the Bolometer (Channels 15+18 for further reference) and the Soft X-Ray (SXR, channels 10+12) diagnostic. In the next row down the amplitude of selected differences of magnetic pick up coils is plotted being proportional of $n=1 / n=2$ MHD perturbations in the plasma. From further MHD analysis it has become clear that before $t=8 \mathrm{~s}$ the $\mathrm{n}=1$ contribution is dominated by Fishbone activity and the $n=2$ contribution is dominated by the $n=2$ harmonic of the Fishbones. The high signal during the main heating phase is therefore not showing a danger of disruption. In the next graph a central and an off-axis electron temperature from the High Resolution Thomson Scattering (HRTS) diagnostic is shown. In the bottom row an estimated W concentration 

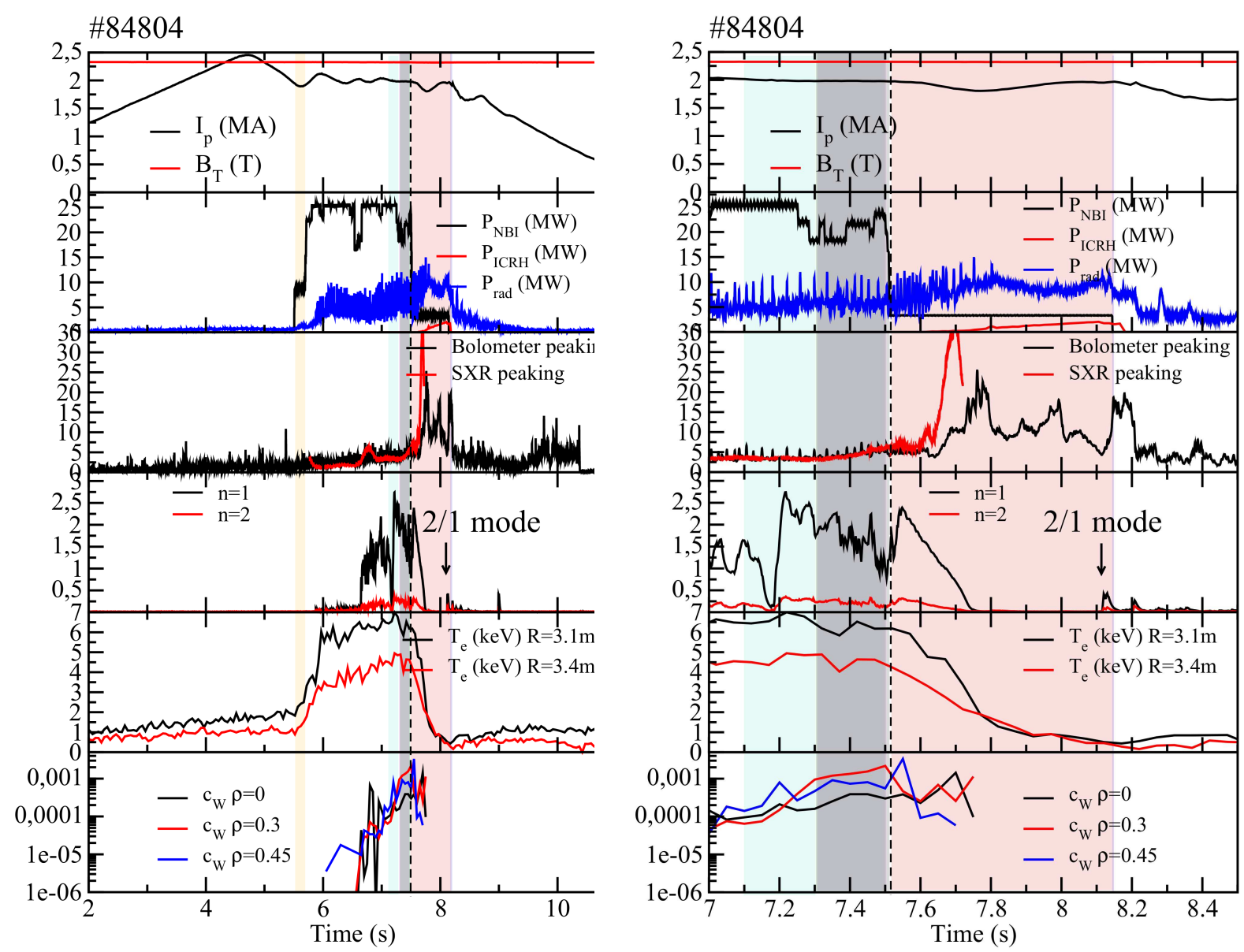

Figure 1. Overview of a typical hybrid pulse termination including the marking of the considered discharge phases as vertical bars on the left. And the same time traces for a reduced time interval on the right. Also the later discussed phases are indicated as colored shadowns: $200 \mathrm{~ms}$ windows at the end of the main heating phase (black), the maximum performance (maximum stored energy, turquese), the maximum SXR radiation peaking within the main heating (same time window as end of heating, pink), the maximum radiation fraction within the main heating phase (same time window as end of heating, green) and at the beginning of the main heating (orange). In addition the low power phase (red) following the main heating. Missing in this pulse is a time window of $0.7 \mathrm{~s}$ at the start of the following Ohmic phase (blue).

from a simple deconvolution of the SXR radiation and atomic data as input [13] is plotted for different radii. The plasma does not accumulate heavy $\mathrm{Z}$ impurities (mainly $\mathrm{W}$ ) in the main heating phase and has only a moderate $\mathrm{W}$ concentration of $10^{-4}$ opposite to the cases discussed in $[14,11]$. At the time when the NBI power reduces at $t=7.5 \mathrm{~s}$ the $\mathrm{W}$ concentration off-axis increases to almost $10^{-3}$ but the radiation profile is not peaked yet and the total radiation is significantly larger than the heating power. $100 \mathrm{~ms}$ later the peaking of the SXR diagnostic increases strongly, which is typical for high $\mathrm{Z}$ impurity accumulation. The electron temperature drops within $500 \mathrm{~ms}$ and the profile flattens. The impurity peaking between $\mathrm{t}=7.7 \mathrm{~s}$ 
and $t=8.2 \mathrm{~s}$ is mainly seen on the bolometer and is increasing again. In figure 2 a tomographic reconstruction of the radiation measured by the bolometer at $t=7.85 \mathrm{~s}$ is shown. Even though there are some vertical and horizontal artifacts the reconstruction shows the strong peaking at about $100 \mathrm{~ms}$ before the onset of the $\mathrm{n}=1$ mode. The estimate of the $\mathrm{W}$ concentration in this phase fails because the plasma temperature becomes too low to detect the SXR radiation through up to $250 \mu \mathrm{m}$ thick Be filters. The peaking factors of the SXR and the bolometer

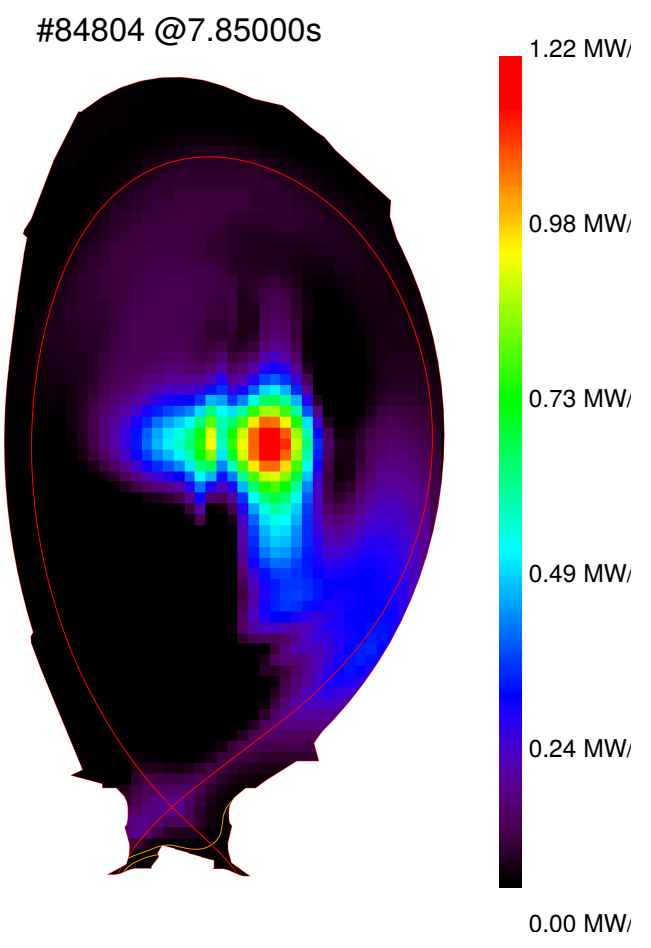

Figure 2. Radiation peaking in a tomographic reconstruction shortly before the MHD mode is excited.

diagnostics are very different in the main heating phase due to a strong $T_{\mathrm{e}}$ dependence in the SXR radiation which is much weaker on the total radiation. The change in $T_{\mathrm{e}}$ profile leads then to the excitement of a different $n=1$ mode which locks shortly afterwards (as reported in [10]). The fact that the amplitude of the $n=1$ signal is small is mainly caused by the quick locking while the mode still grows. As a consequence the control system stops the pulse by initiating a fast current ramp. In the statistics described later this pulse counts as a locked mode in the low power phase.

The excitement of the $\mathrm{n}=1$ mode is connected to a change in $q$-profile (as reported in [10]). In figure 3 on the left hand side the $q$-profile development for a pulse with locked mode (black) and without locked mode (red) is shown. A different pair of pulses is shown here because the MSE measurement in the low power tail is often not available because the measurement beam is in most pulses switched off. On the right hand side the electron temperatures are plotted. With the switch off of the main heating the temperature drops in both cases strongly. In the case without locked mode some temperature peaking remains 
whereas in the other case very flat temperature profiles are found. In this phase, external q-profile development in hybrid landing
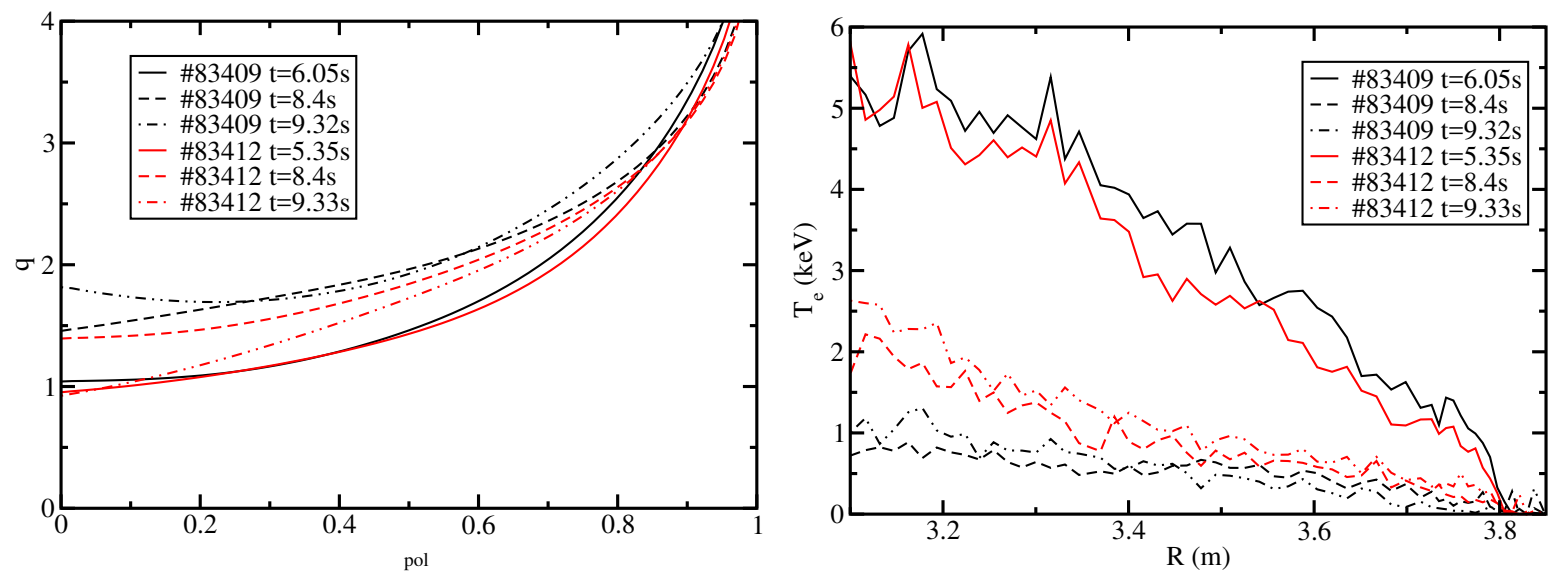

Figure 3. $q$-profile and $T_{\mathrm{e}}$-profile development with (black) and without (red) locked mode

current drive is negligible and the current profile is dominated by the Ohmic contribution. Since the temperatures are low the $q$-profile is changing on a fast time scale reflecting the changes in the electron temperature profile. The $q$-profile in the pulse without locked mode somewhat recovers and stays monotonic, in the pulse with locked mode a high $q_{\min }$ can be seen and also shear reversal is found in the central region. To assess the tearing mode stability the toroidal plasma current density gradient and the resistivity at the $q=2$ surface for both plasmas have been computed. In the case where a mode occurs the resistivity is larger and an increase in current density gradient is observed before the mode is triggered. In the case without mode locking the resistivity is generally lower and no sustained current density gradient increase is observed. Qualitatively this supports that the plasma which develops a locked mode was more tearing unstable before than the plasma without. In addition the rotation is usually low as well and a locking is likely. In the pulse discussed earlier (figure 1) the electron temperature recovers after the mode locking and the radiation peaking reduces before a disruption could occur. In about 53\% of the hybrid pulses a ramp down succeeds after a locked mode without disruption (and of course many plasmas terminate without locked mode or disruption). Compared to the pulses with lower $q_{95}$ in the database the rate is lower. Baseline plasmas outside of this database are usually run at higher plasma current where the use of the disruption mitigation valve is mandatory and therefore this statistics can't be made. The higher current does also change other properties of the termination which may make it more difficult to land cleanly. It is often observed that the plasma density increases with increasing plasma current. As a consequence the density decay at the end of the H-mode phase becomes more important (as discussed in [12]). Another consequence at JET is the reduced NBI penetration already during the main heating and therefore a lower power density in the centre which makes the plasma less stable against high $\mathrm{Z}$ impurity accumulation. On the other hand the baseline plasmas usually do start the normal termination with less impurities in the core because the $\mathrm{W}$ sources during the main heating phase are better controlled with the caveat of a reduced $H_{98, y 2}$ factor. As shown in [10] high $\mathrm{Z}$ impurity accumulation is still the most important parameter but in addition the reduced MHD stability and the more difficult 
density reduction add complexity for JET. For ITER the reduced capability of shape control and reduced vertical position control add further complexity.

\section{Database}

For this paper a database is developed in order to reveal the intricate relationship between various plasma parameters at different times in the discharge affecting the termination of the plasma. The database consists of 480 pulses (all hybrid pulses in the ILW which reached the main heating phase) of which 215 pulses had a locked mode and 101 of them disrupted (5 in the main heating phase due to a stop of vertical position control and 2 in the main heating phase due to excessive radiation and 76 in the Ohmic phase after the heating has stopped, the Ohmic phase before the heating starts is not considered here). In the 40 pulses with $q_{95}<3.6$ ( 3.8 is the nominal value for a JET hybrid pulse) 33 had a locked mode and 18 disrupted. In the reduced database (pulses with ILW until 2014 [11]) 63\% of the pulses had a locked mode whereas the extended database (all pulses with ILW until now) has now 45\% locked modes which indicates a learning effect taken place in the years in between. On the other hand the disruptivity in the 2014 database was $13 \%$ and in the extended database it has increased to

Radiation peaking in ohmic phase

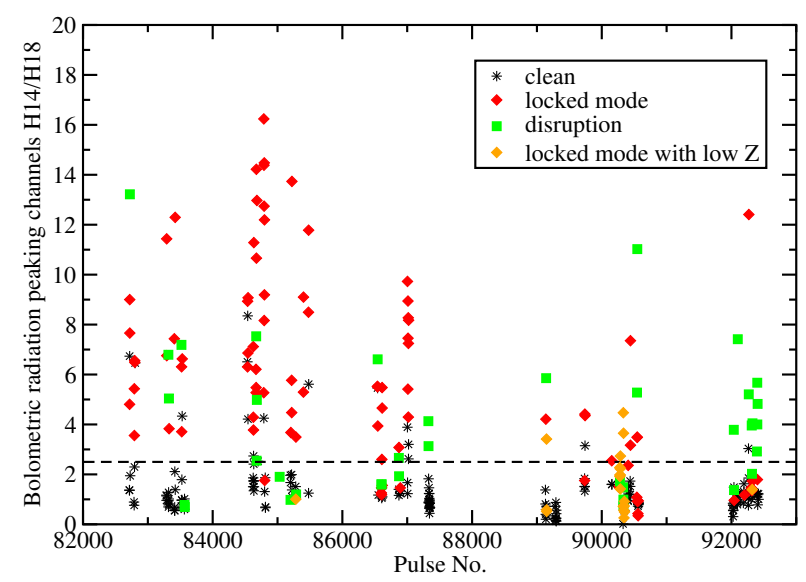

Figure 4. Bolometric radiation peaking in Ohmic phase - definition of threshold

$21 \%$. The increase in disruption rate is mainly because of the higher currents used in the more recent experiments. Above a plasma current of 2MA a triggering of the disruption mitigation valve (DMV) is mandatory if a disruption is predicted, e.g. if the locked mode signal is exceeding a certain value. This protection has been used also at smaller currents than $2 \mathrm{MA}$ in order to prepare the scenario. Out of the 49 disruptions 30 disruptions were actually caused by the firing of the DMV. Whether the plasma would have disrupted without DMV or not cannot be determined. Since before 2015 the operation was different and the DMV in these cases was triggered only during an already ongoing disruption the statistics is misleading. An interesting subset of the pulses have been seeded by Neon, either to reduce the power load in the divertor or to improve the ion temperature measurement. Of the 29 pulses with Neon, 28 pulses have 
a locked mode and 8 of those disrupt. The only plasma which terminates cleanly is the one with the lowest Neon gas puff. The main difference in the parameters during the termination is that pulses with Neon in the main heating phase tend to maintain a higher density during the low power and Ohmic phase which then during the current ramp down causes either a density limit disruption as often observed during operation in a carbon machine or results in a locked mode but without disruption. A connection to the density peaking observed when Neon is seeded as reported in [15] is possible. The plasmas start already at higher densities at the beginning of the termination. From now on only the extended database is considered. To understand the importance of the different phases of the pulse a set of 54 parameters has been collected in 7 phases of the pulse. The phases are defined as $200 \mathrm{~ms}$ windows at the end of the main heating phase, the maximum performance (maximum stored energy), the maximum SXR radiation peaking within the main heating, the maximum radiation fraction within the main heating phase and at the beginning of the main heating. In addition the low power phase following the main heating and a time window of $0.7 \mathrm{~s}$ at the start of the following Ohmic phase are taken, both cut short in case of a locked mode or disruption. Part of the phases are also indicated in figure 1 and in figure 8 as colored bars. The phases can be overlapping, e.g. in figure 1 the phase of the maximum radiation and the maximum radiation peaking are the same time, or be missing because a locked mode occurs before. In figure 1 no ohmic termination phase is considered because the low power phase already results in a locked mode. From this point on the discharge is controlled by a scenario independent stop procedure. The pulses in the database are collected over several years with changing boundary conditions. Changes are the possible divertor configurations, the oldest pulses were done on a horizontal tile only, in order not to damage the coated tiles close to the pump duct and the vertical target. This period was followed by the exploration of the tiles close to the pump duct for the main heating phase and the termination phase. Lately the termination phase has been moved to the vertical target to avoid stops due to high surface temperatures on the tiles close to the pump duct. A correlation of the radiation peaking to the divertor geometry has not been attempted, also because it strongly determined by the time when the pulse has been done. In order to make use of the collected data a common reference point has to be found. In figure 4 the bolometric peaking factor is shown, the pulses with locked modes have red symbols and clean plasmas black ones. Above a peaking of 2.5 we find $75.3 \%$ of the pulses which have a locked mode not taking into account pulses with Neon injection and $62 \%$ of the pulses which disrupted but only $9.6 \%$ of the pulses without problems. This shows that in most pulses the radiation peaking in the Ohmic phase is a critical parameter. Doing the exercise with the other parameters in the database does not yield an as good discrimination criteria and therefore the radiation peaking in the Ohmic phase has been used as a reference for a correlation analysis with other parameters to get an indication of their importance. As can be seen in figure 6 the radiation peaking in different phases remains an important parameter in this analysis. Together with the physics picture given in the introduction this is all consistent and is indicating that the statistics not only gives correlations but shows causality in this case. 


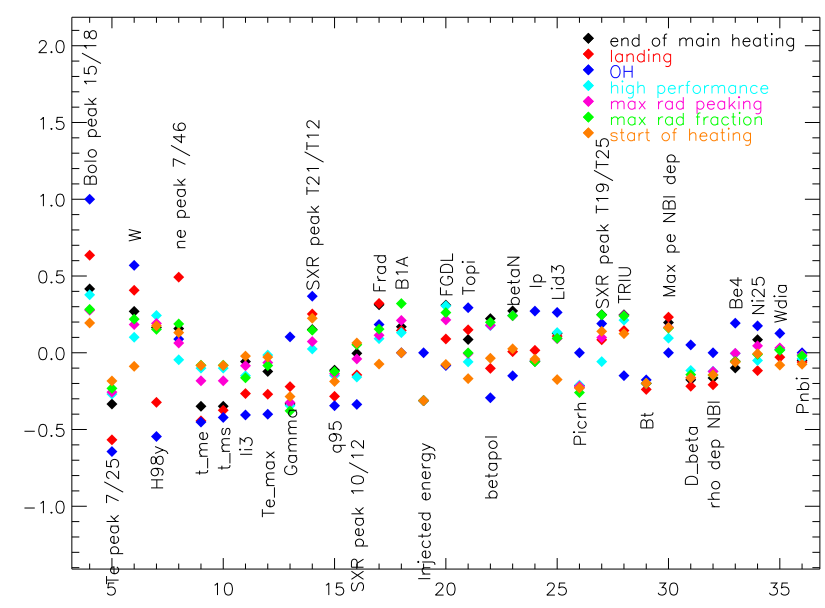

Figure 5. Correlation coefficient of bolometric radiation peaking during the Ohmic phase plotted against reduced set of database parameters. X-axis sorted according to highest correlation coefficients ignoring the different phases.

\section{Analysis of the database using correlations}

In the following a correlation analysis between different parameters within the database is presented. The correlation analysis tests essentially how well the functional dependence of two arbitrary data sets can be described by a linear dependence. The Pearson correlation coefficient e.g. between the heating power in the main heating phase and the radiation peaking in the Ohmic phase of all pulses in the database is calculated. The idea behind this analysis is that first the most important parameters are identified and second some information on the temporal evolution is obtained. To make an example, if bad terminations would be caused predominantly by large impurity events in the main heating phase, then a large correlation coefficient between the maximum radiation and/or maximum radiation fraction in the high radiation fraction time window, and the radiation peaking in the Ohmic phase should be visible - which is not the case. The correlation coefficient of a reduced set of parameters is shown in figure 5. The colours symbolise the different phases (also indicated in figures 1 and 8) and the x-axis is sorted from higher correlations on the left to lower on the right using the absolute values and not the phase specific ones.

The signals are described in table 1. In this table there are 1 bolometric plus 3 SXR (Soft $\mathrm{X}$-Ray) peaking factors which all are related to impurity radiation peaking. The fact that $\mathrm{W}$ has a complicated spatial and temporal evolution (see [14]) makes a 0-D analysis complicated as well. First, after an influx, $\mathrm{W}$ is usually not accumulating in the core immediately, but larger $\mathrm{W}$ concentrations are observed off-axis at about half radius with relatively large poloidal asymmetries due to the large toroidal rotation speed. In this case a horizontal channel in the mid-plane will observe an increase in radiation but a vertical channel from a top view not. So the peaking of the horizontal channels detects an increase in the $\mathrm{W}$ concentration but not necessarily a central peaking. On the other hand the signal of the vertical camera channels will see just the central peaking of the impurities but only if the Shafranov shift is right. Therefore 
Table 1. Summary table of most important correlations in all seven phases.

\begin{tabular}{|c|c|}
\hline Label & Description \\
\hline Bolo peak $15 / 18$ & $\begin{array}{l}\text { ratio of two horizontal bolometer channels, one through the centre } \\
\text { and the other cutting at about half radius }\end{array}$ \\
\hline Te peak $7 / 25$ & $\begin{array}{l}\text { electron temperature ratio from the HRTS diagnostic using } \\
\text { a channel at } 3.1 \mathrm{~m} \text { (central) and at } 3.4 \mathrm{~m} \text { (off-axis) }\end{array}$ \\
\hline $\mathrm{W}$ & W source signal (WII) in the divertor measured by a VUV spectrometer \\
\hline H98y & $\begin{array}{l}\text { H-mode scaling factor describing the confinement relative to the } \\
\text { IPB } H^{98 y 2} \text { scaling }\end{array}$ \\
\hline ne peak $7 / 25$ & electron density channel ratio (as for $T_{\mathrm{e}}$ ) from the HRTS diagnostic \\
\hline t_me/t_ms & ending/starting time of the window where the data have been taken \\
\hline li3 & internal inductance of the plasma \\
\hline Te_max & highest electron temperature measured in the plasma by HRTS \\
\hline Gamma & total gas flux from the fuelling system \\
\hline SXR peak T21/T12 & ratio of 2 vertical lines of sight, one through the center and one going off-axis \\
\hline SXR peak T19/T25 & similar as SXR peak T21/T12 slightly different positions \\
\hline SXR $10 / 12$ & ratio of horizontal channels with thicker Be-filters \\
\hline q95 & $q_{95}$ from the equilibrium reconstruction \\
\hline Frad & radiated power measured by bolometer divided by the total heating power \\
\hline B1A & amplitude of the strongest $n=1$ mode in the plasma \\
\hline injected energy & time integral of the heating power over the duration of the pulse \\
\hline FGDL & electron density divided by the Greenwald scaling density \\
\hline Topi & totally radiated power from the bolometer \\
\hline betapol & $\begin{array}{l}\text { poloidal beta calculated by the equilibrium reconstruction } \\
\text { Def.: } \beta_{\mathrm{pol}}=2 \mu_{0} \frac{\langle p\rangle_{A}}{\left\langle B_{\mathrm{pol}} \text { rangle }\right.} \text {, where }\langle p\rangle_{A} \text { is the poloidal cross-section averaged } \\
\text { plasma pressure and }\left\langle B_{\mathrm{pol}}\right\rangle \text { the average poloidal magnetic field on the plasma } \\
\text { boundary }\end{array}$ \\
\hline betaN & normalised beta, $\beta_{N}=\beta_{\text {tor }} / \frac{I_{P}}{a B_{T}}$ with $B_{T}$ toroidal magnetic field \\
\hline Lid3 & line integrated density through the centre from the interferometer diagnostic \\
\hline Picrh & injected power from the ICRH heating \\
\hline TRIU & $\begin{array}{l}\text { upper triangularity of the plasma configuration calculated by the } \\
\text { equilibrium reconstruction }\end{array}$ \\
\hline Max pe NBI dep & $\begin{array}{l}\text { maximum power density deposited in the electrons by the NBI heating } \\
\text { calculated by the inter-shot PENCIL }\end{array}$ \\
\hline rho dep NBI & radius of the maximum power deposition from PENCIL \\
\hline $\mathrm{Be} 4$ & calibrated line intensity from a VUV spectrometer proportional to the Be source \\
\hline NI25 & proportional to the Nickel concentration measured as well by a VUV spectrometer \\
\hline Wdia & total stored energy from diamagnetic loop \\
\hline Pnbi & total injected power from the neutral beam heating system \\
\hline
\end{tabular}




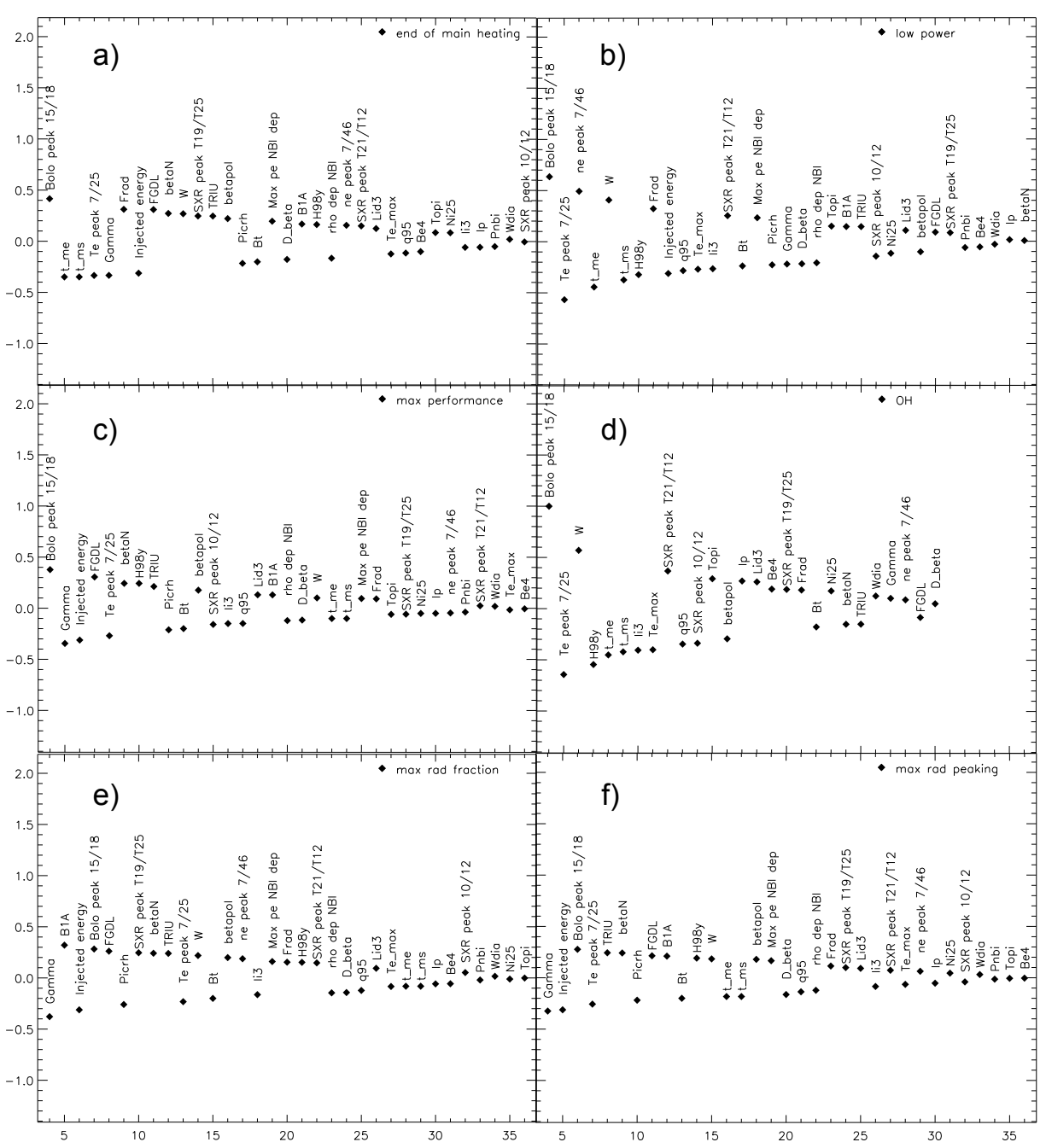

Figure 6. Correlation coefficient in different phases of the discharges.In subfigure a) data in the time interval of the last $200 \mathrm{~ms}$ before the auxiliary heating power for the termination is reduced is averaged. In subfigure b) all data in the low power phase at the start of the termination phase is averaged and analysed. Parts c),e),f) are values averaged over $200 \mathrm{~ms}$ around the time point of the maximum stored energy, maximum radiation fraction and maximum radiation peaking within the analysed discharge. In subfigure d) the average of the first 700ms (or until a locked mode occurs) of the final ohmic phase are analysed. The $\mathrm{x}$-axis sorted according to importance in the labelled phase of the pulse. 
several different ratios of vertical channels are necessary to detect the peaking for different Shafranov shifts e.g. during the main heating phase and during the low power phase during the termination.

Table 2. Summary table of most important correlations to the radiation peaking in the Ohmic phase for all seven phases.

\begin{tabular}{|c|c|c|c|c|c|c|}
\hline & Max pos & 2nd & $3 \mathrm{rd}$ & Max neg & 2nd & $3 \mathrm{rd}$ \\
\hline All & $\begin{array}{l}\text { Rad peak } \\
(0.64) \text { low pow. }\end{array}$ & $\begin{array}{l}\text { W influx } \\
(0.57) \mathrm{OH}\end{array}$ & $\begin{array}{l}n_{\mathrm{e}} \text { peak. }(0.49) \\
\text { low power }\end{array}$ & $\begin{array}{l}T_{\mathrm{e}} \text { peaking } \\
(-0.65) \mathrm{OH}\end{array}$ & $\begin{array}{l}H_{\mathrm{y}, 2}^{98}(-0.54) \\
\mathrm{OH}\end{array}$ & $\begin{array}{l}l_{i}(3) \\
(-0.4) \mathrm{OH}\end{array}$ \\
\hline $\begin{array}{l}\text { End of } \\
\text { heating }\end{array}$ & $\begin{array}{l}\text { Rad. peak } \\
(0.42)\end{array}$ & $\begin{array}{l}f_{\mathrm{rad}} \\
(0.31)\end{array}$ & $\begin{array}{l}f^{\mathrm{GW}} \\
(0.31)\end{array}$ & $\begin{array}{l}T_{\text {e peaking }} \\
(-0.33)\end{array}$ & $\begin{array}{l}\Gamma \\
(-0.33)\end{array}$ & $\begin{array}{l}\text { Injected En. } \\
(-0.33)\end{array}$ \\
\hline $\begin{array}{l}\text { Low } \\
\text { power }\end{array}$ & $\begin{array}{l}\text { Rad. peak } \\
(0.64)\end{array}$ & $\begin{array}{l}n_{\mathrm{e}} \text { peaking } \\
(0.49)\end{array}$ & $\begin{array}{l}\text { W influx } \\
(0.41)\end{array}$ & $\begin{array}{l}T_{\text {e peaking }} \\
(-0.57)\end{array}$ & $\begin{array}{l}H_{\mathrm{y}, 2}^{98} \\
(-0.32)\end{array}$ & $\begin{array}{l}\text { Injected En. } \\
(-0.31)\end{array}$ \\
\hline $\mathrm{OH}$ & $\begin{array}{l}\text { W influx } \\
(0.57)\end{array}$ & $\begin{array}{l}\text { SXR peak. } \\
(0.37)\end{array}$ & $\begin{array}{l}P_{\mathrm{rad}} \\
(0.29)\end{array}$ & $\begin{array}{l}T_{\text {e peaking }} \\
(-0.65)\end{array}$ & $\begin{array}{l}H_{\mathrm{y}, 2}^{98} \\
(-0.54)\end{array}$ & $\begin{array}{l}l_{i}(3) \\
(-0.4)\end{array}$ \\
\hline $\begin{array}{l}\text { Max. } \\
\text { perf. }\end{array}$ & $\begin{array}{l}\text { Rad. peak } \\
(0.38)\end{array}$ & $\begin{array}{l}f^{\mathrm{GW}} \\
(0.31)\end{array}$ & $\begin{array}{l}\beta_{\mathrm{N}} \\
(0.24) \\
\end{array}$ & $\begin{array}{l}\Gamma \\
(-0.34) \\
\end{array}$ & $\begin{array}{l}\text { Injected En. } \\
(-0.31)\end{array}$ & $\begin{array}{l}T_{\text {e }} \text { peaking } \\
(-0.27)\end{array}$ \\
\hline $\begin{array}{l}\text { Max. rad. } \\
\text { peaking }\end{array}$ & $\begin{array}{l}\text { Rad. peak } \\
(0.28)\end{array}$ & $\begin{array}{l}\delta^{\mathrm{U}} \\
(0.24)\end{array}$ & $\begin{array}{l}\beta_{\mathrm{N}} \\
(0.24)\end{array}$ & $\begin{array}{l}\Gamma \\
(-0.32)\end{array}$ & $\begin{array}{l}\text { Injected En. } \\
(-0.31)\end{array}$ & $\begin{array}{l}T_{\text {e peaking }} \\
(-0.27)\end{array}$ \\
\hline $\begin{array}{l}\text { Max. rad. } \\
\text { fraction }\end{array}$ & $\begin{array}{l}\text { B1A } \\
(0.32)\end{array}$ & $\begin{array}{l}\text { Rad. peak } \\
(0.28)\end{array}$ & $\begin{array}{l}f^{\mathrm{GW}} \\
(0.26)\end{array}$ & $\begin{array}{l}\Gamma \\
(-0.38)\end{array}$ & $\begin{array}{l}\text { Injected En. } \\
(-0.31)\end{array}$ & $\begin{array}{l}P_{\text {ICRH }} \\
(-0.26)\end{array}$ \\
\hline $\begin{array}{l}\text { H-mode } \\
\text { entrance }\end{array}$ & $\begin{array}{l}\text { SXR peak. } \\
(0.22)\end{array}$ & $\begin{array}{l}\text { Rad. peak } \\
(0.19)\end{array}$ & $\begin{array}{l}H_{\mathrm{y}, 2}^{98} \\
(0.18)\end{array}$ & $\begin{array}{l}\text { Injected En. } \\
(-0.31)\end{array}$ & $\begin{array}{l}\Gamma \\
(-0.29)\end{array}$ & $\begin{array}{l}P_{\text {ICRH }} \\
(-0.23)\end{array}$ \\
\hline
\end{tabular}

The different sensitivity for high $\mathrm{Z}$ impurities in the SXR diagnostic compared to the total radiation makes it useful to look to it separately. The geometrical properties are as discussed before. In addition the SXR diagnostic is strongly temperature dependent but very sensitive to high $\mathrm{Z}$ impurities. Therefore the diagnostic is ideal to diagnose a $\mathrm{W}$ accumulation in the main heating phase but almost without use in an Ohmic phase due to the lower temperatures. Additionally the horizontal SXR camera has thicker Be-filters (350 $\mu \mathrm{m}$ compared to $250 \mu \mathrm{m}$ in the vertical camera) and measures generally only at higher temperatures (this explains the anti correlation in the Ohmic phase).

The magnetic fluctuation signals are from magnetic probes. B1A is the amplitude of the strongest $\mathrm{n}=1$ mode in the plasma which is usually produced by either sawteeth or fishbones, $2 / 1$ modes are extremely rare in the JET hybrid mode plasmas. Unfortunately the $n=2$ and $\mathrm{n}=3$ signals were so scarcely available for the database that they had to be omitted.

The database contains 18 more signals which have been omitted in the figures either because they are redundant (4 bolometer peakings, 2 Te peaking factors, 5 SXR peaking factors, the thermal confinement time, line integrated density off-axis, density peaking factor from the interferometer channels, total radiated power from the bolometer using a different integration method) or because they are not well populated as the $n=2$ and $n=3$ MHD 
amplitudes.

The highest correlations are seen with the low power phase and within the Ohmic phase itself. In figure 6 a-f the same data as in figure 5 but reduced to the appropriate phase and sorted just on the data shown are plotted. In general there are two different sets of important data depending on the phase. In case of a low power tail or Ohmic phase the most important parameters are connected to the electron temperature/confinement, radiation and $\mathrm{W}$ source as can be seen in figure 6-b and 6-d. An interesting exception here is the significant dependence on the density peaking in the low power phase which has only become visible in the extended data set. In the neoclassical transport analysis e.g. in [14] the density gradient is the driving term for heavy $\mathrm{Z}$ impurity accumulation. During any of the high power phases before the initiation of the termination, the radiation peaking, the gas flux, the injected energy and the electron temperature peaking are main players. In general the further away from the Ohmic phase the data are taken the lower the correlation to the Ohmic phase is. This sounds trivial but in the end it means that the plasma does not have a long memory for events and the quality of the termination is mainly determined by the design of the termination phase. But the remaining importance of radiation and temperature peaking shows that it is easier to land a healthy discharge than a discharge which already is impurity accumulated.

In table 2 the three most positive and three most negative correlations for all 7 phases are summarised. The data have been selected to reduce the number of strongly related parameters. Not explicitly in the database are parameters like ELM size and frequency. These parameters might be important as shown in the baseline analysis [16]. Detailed analysis will be scope of future work. For the time being only the parameters which we think are important in determining the ELM frequency like heating power, gas fuelling, $q_{95}$ and radiation power are part of the database.

\section{Proposed new termination scenario}

The data gathered in this database indicates that events which happen closer to the termination of the plasma are more important than events earlier - or in other words by choosing the right termination we might be able to repair damage which has occurred earlier, e.g. by impurity events, MHD or generally impurity accumulation. On the other hand there are some examples in the database where the plasma during the main heating phase is quite healthy but still develops a locked mode later due to a wrong termination.

Based on this assumption and the main findings summarised in table 2 a revised termination scenario has been proposed and partially explored. The main parameters are connected to the radiation losses under conditions of a significant $\mathrm{W}$ concentration in the plasma core and a sudden power reduction together with significant $\mathrm{W}$ sources. Therefore as a first step the heating power is reduced compared to the main heating phase while keeping or increasing the ICRH power. The remaining power has to provide enough absorbed power in the centre to compensate the radiation losses. The power reduction can probably not be strong enough on JET to go out of H-mode. At the same time the gas fuelling is increased and the strike points are moved onto the vertical target to reduce the $\mathrm{W}$ sources. The vertical target is 

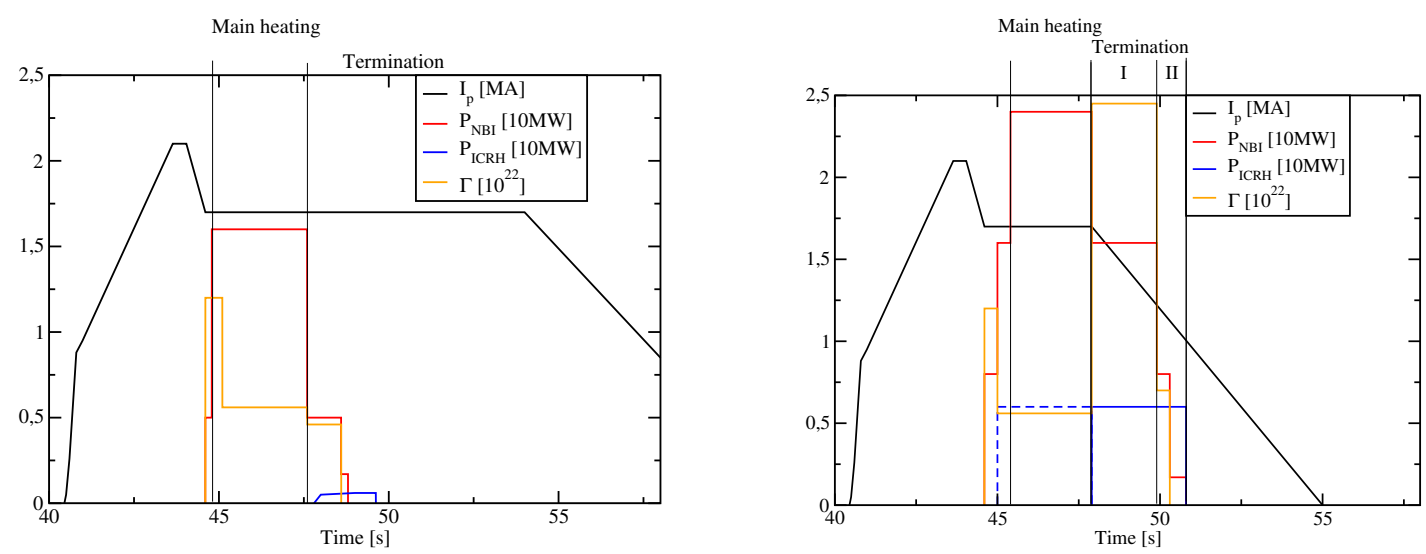

Figure 7. "Traditional" termination scheme on the left (before 2014) and suggested new termination scheme on the right (partially tried in 2015/16)

chosen to avoid overheating of the divertor target used and the high gas fuelling reduces the temperature and increases the ELM frequency which has been identified as critical parameter in baseline discharges [16]. The high central power density prevents a flattening of the temperature profile. In addition the lowered input power and increased gas flux will reduce the energy confinement time. The second step would reduce the NBI heating power and the gas fuelling drastically and keeps the ICRH power on. In this phase the density and the W source should reduce while the central heating is supposed to control the impurity peaking. As a last step the Ohmic phase will be at zero feed forward gas fuelling but density feedback control set to a low density to avoid error field locked modes, density limit disruptions and a strong temperature reduction. The plasma current would be ramped down during all termination phases to reduce the chance of an existing locked mode to trigger a disruption and generally to reduce the energy in the plasma and the possible disruption forces.

The new termination scheme was partially tried in the campaigns $2015 / 2016$. But it was not always possible to stick to the described scheme for technical reasons. Therefore the free parameters like the length of termination phase, the remaining heating power or the right gas level could not be optimised. Nevertheless the locked mode ratio has been reduced from $63 \%$ to $45 \%$. Even though higher current and higher heating power plasmas were run. It was felt more difficult to terminate the higher current plasmas cleanly even though this difficulty is not visible in the statistics. An example of a new termination is shown in figure 8. The figure is organised as figure 1. The example chosen here is at the highest current of $I_{\mathrm{P}}=2.5 \mathrm{MA}$ which has been used so far in the hybrid development at the nominal $q_{95}$. The radiation fraction is already much higher in the main heating phase compared to the example discussed earlier. The SXR peaking factor is above 10 already at $t=8 \mathrm{~s}$ and has a strong first peaking at about $t=9.5 \mathrm{~s}$ caused by an impurity influx (can be seen on the radiation power) and a loss of regular ELMs at $t=9 \mathrm{~s}$. At about $t=10 \mathrm{~s}$ another impurity influx with a subsequent strong peaking phase is visible. As can be seen in row 6 on the $\mathrm{W}$ concentration a strong ( few times $10^{-3}$ ) off-axis enrichment at about half radius follows shortly afterwards. The NBI power is partially lost at $t=10.5 \mathrm{~s}$ which is very similar to the proposed termination scenario but the 


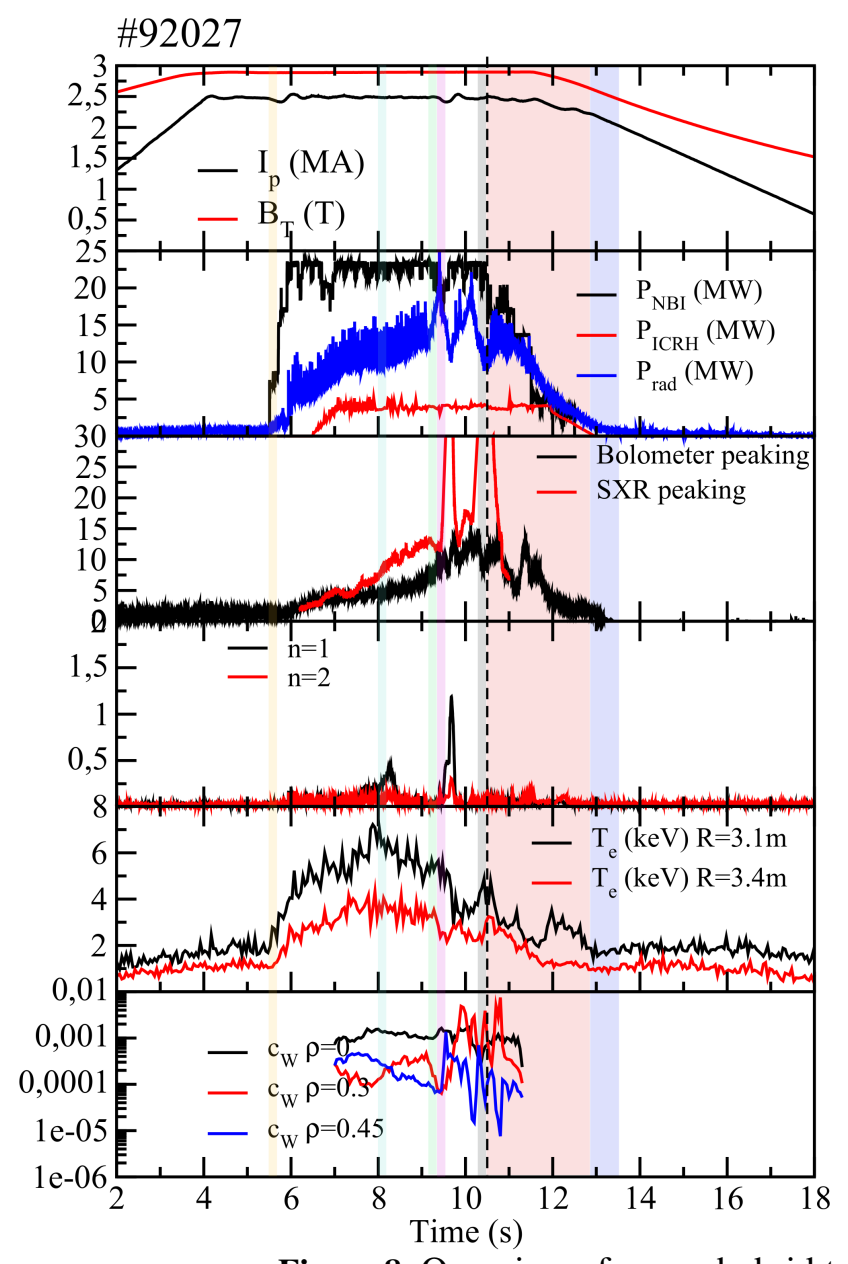

Figure 8. Overview of a new hybrid termination

current ramp and the strike point position change happen only at about $t=12 \mathrm{~s}$. Differently to the example discussed in the introduction here the radiation stays mostly below the applied heating power and the bolometric radiation peaking decays towards the low power phase. The ICRH heating is kept at high power levels until $t=12 \mathrm{~s}$. The gas injection is controlled in a real time feedback on the ELM frequency and increases at $t=10.3 \mathrm{~s}$ by $100 \%$. In this case the radiation peaking reduces before the heating power is switched off and the electron temperature profile remains peaked. No $n=1$ mode is excited and therefore no locking is observed. The increase of the electron temperature at $t=12 \mathrm{~s}$ leads also to an increase in SXR signal. At the same time the plotted SXR peaking and the $\mathrm{W}$ concentrations are increasing without an obvious reason. This indicates that the measurement between $t=11 \mathrm{~s}$ and $t=12 \mathrm{~s}$ is probably not trustworthy and the SXR peaking and the $\mathrm{W}$ concentrations are still large in this time interval. 


\section{Summary and conclusion}

A novel way of analysing the termination of JET hybrid plasmas is presented. The first finding is that the occurrence of locked modes and with about $50 \%$ chance a disruption is connected to an increased radiation fraction after reducing the heating power. After switching off the heating power completely a radiation peaking in the Ohmic phase leads to a flattening or reversing of the temperature profile which in turn leads to an increase in central $q$ and to shear reversal. Next a 2/1 MHD mode is triggered and most often locks shortly afterwards. In the created database a threshold value for the radiation peaking in the Ohmic phase can be given above which the probability of generating a locked mode is larger than $75 \%$. The radiation peaking in the Ohmic phase is the best parameter in this database linked to the occurrence of locked modes. It is therefore used as a reference for a correlation analysis with 54 parameters in 7 phases of the discharge. The correlation analysis highlights parameters as most important which are consistent with the picture of a radiation driven $q$-profile change as the dominating effect to generate locked modes. It shows as well that the parameters closer in time to the start of the termination are having higher correlation coefficients and therefore are more important. This indicates that a good termination procedure might be able to terminate pulses safely even if they are not healthy during the main heating phase. A new termination procedure has been proposed and partially implemented. As a measure of success the locked mode probability has reduced from $63 \%$ to $45 \%$ afterwards. Not understood in this context is that the disruption probability has increased from $13 \%$ to $21 \%$. But the fact that the disruption mitigation valve in the newer pulses is triggered more aggressively makes the disruption statistics not comparable. The termination procedure is not yet optimised and further improvements are possible.

\section{Acknowledgement}

This work has been carried out within the framework of the EUROfusion Consortium and has received funding from the Euratom research and training programme 2014-2018 under grant agreement No 633053. The views and opinions expressed herein do not necessarily reflect those of the European Commission or the ITER Organization.

\section{References}

[1] Hobirk J et al. 2012 PPCF 54095001

[2] Gruber O et al. 1999 Phys. Rev. Lett. 831787

[3] Wolf R C et al. 1999 Plasma Phys. Control. Fusion 41 B93

[4] Staebler A et al. 2005 Nucl. Fusion 45617

[5] Luce T C et al. 2001 Nucl. Fusion 411585

[6] Luce T C et al. 2004 Physics of Plasmas 112627

[7] Wade M R et al. 2005 Nucl. Fusion 45407

[8] Sips A C C et al. 2007 Nucl. Fusion 471485

[9] Joffrin E et al. in Fusion Energy 2008 (Proc. 22nd Int. Conf. Geneva, 2008) (Vienna: IAEA) CD-ROM file Geneva and http://www-naweb.iaea.org/napc/physics/FEC/FEC2008/html/index.htm

[10] de Vries P C et al. 2014 PoP 21056101 
[11] Hobirk J et al. 2014 Europhysics Conference Abstracts, Proc. 41st EPS Conference Vol. 38F P1.003 (Berlin)

[12] de Vries P C et al. 2018 Nucl. Fusion 58026019

[13] Pütterich T et al. 2013 PPCF 5512

[14] Angioni C et al. 2014 NF 54083028

[15] Challis C D et al. 2017 Europhysics Conference Abstracts, Proc. 44st EPS Conference Vol. 41F P2.153 (Belfast)

[16] Köchl F et al.,Evolution and control of tungsten transport in the termination phase of JET H-mode discharges and implications for ITER. Preprint: 2016 IAEA Fusion Energy Conference, Kyoto [EX/P614] 